\title{
Uninformed consent?
}

\author{
The US should revamp rules on informed consent to ensure that people have all of the information and support they \\ need before deciding to enroll in clinical trials.
}

W

hen people decide to take part in a clinical study, they do so in the hopes that the experimental treatment will help them in some way. But in some cases, unforeseeable complications can make the therapy toxic or even fatal.

An apparent example was Jolee Mohr, a 36-year-old woman who enrolled in a phase $1 / 2$ gene therapy trial for her arthritis. Mohr received the second shot of the investigational treatment on 2 July and died of multiple organ failure 22 days later.

Under current US rules, before people agree to take part in a clinical trial, they must fully understand what benefits or risks they can expect from the experimental therapy. But Mohr's husband claims that the risks were not adequately explained to her. He says, for instance, that she expected to benefit from the trial-when in fact the study was only designed to test safety (see page 1008).

If that's true, the US rules covering informed consent and the support network to aid in an individual's decision making may have been inadequate in Mohr's case-and perhaps in scores of others.

These rules should be changed to guarantee that people fully understand what they are getting themselves into when they agree to participate in a clinical study.

The US requires that clinical trial recruits learn why the study is being conducted, how long it might take, what the therapy will entail and whom to contact if there are any questions about the research or about the rights of research subjects. People must also recognize that their participation in the trial is fully voluntary and must be made aware of available alternative treatments.

Generally, trial participants receive an 'informed consent' document providing information about the trial, which, when signed, indicates their understanding of the trial and their consent to participate. But trial recruits may still sign the document without fully comprehending the trial or its potential risks and benefits. In fact, a 2003 study in the journal Cancer showed that individuals often overestimate the clinical benefit they may receive from an experimental therapy.

This was apparently true in Mohr's case. Although the informed consent document she signed identifies some of the risks that may be associated with the therapy, these statements only begin on page 7 of the 13-page document. And despite the requirement that information about the trial be presented to the patient in understandable language, the technical nature of the gene therapy trial may have made the consent document too difficult for Mohr-or indeed most laypeople-to comprehend.

Mohr's consent document was approved by Western Institutional Review Board, a for-profit ethics board hired by Targeted Genetics, the Seattle-based company running the trial. This raises a potential conflict of interest, since for-hire review boards may be tempted to rubber-stamp the consent document in exchange for more business from the company.

To avoid such conflicts, the Office for Human Research Protections, the division of the US government responsible for clinical trial subject safety, should itself convene review boards to evaluate informed consent documents and procedures. The members of these boards should be employed directly by this office.

The rules also mandate that the circumstances under which consent is obtained must "minimize the possibility of coercion or undue influence.” But Mohr's own rheumatologist, Robert Trapp, who arguably may have held 'undue influence' over her in his role as her physician, recruited her to take part in the study. The US National Institutes of Health recommends that doctors should not recruit their own patients into a trial. In cases where this cannot be avoided, the agency recommends that an impartial third party should discuss the research with the trial recruit. These recommendations should be made into law.

Because most individuals in clinical trials are not medical experts and might even lack very basic scientific knowledge, it may be too difficult for them to be able to weigh the risks and benefits of a potential therapy on their own. Therefore, the procedure of discussing the trial with an impartial physician before consenting to join should also be implemented more broadly, particularly in situations where the therapy may be especially risky, or when the individual's condition is not life threatening. Although this may increase the cost of some clinical trials, the cost would certainly be worth the added benefit to the trial's participants.

The involvement of an impartial third party in the decision-making process may be particularly valuable when the doctor responsible for patient recruitment has a financial interest in the study. Trapp's clinic received payment from Targeted Genetics when he brought in trial participants. According to Mohr's husband, she had not known about Trapp's financial interests in the study, and the current rules do not require disclosure of such interests to the patient. This is crucial information that should be made available when individuals are deciding whether to enroll in a trial.

The lack of stringent rules governing informed consent keeps trial participants in the dark about the risks the experimental treatment may pose. And the lack of sufficient oversight by review boards - who may not fully consider the interests of the patients—could only make matters worse. The rules on informed consent need an overhaulbefore the death of another uninformed participant keeps people away from clinical trials for good. 